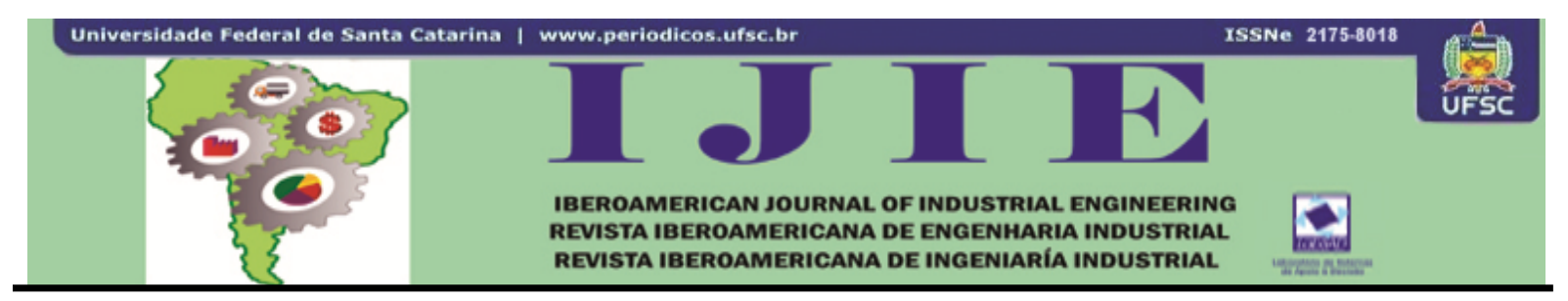

\title{
ESTRATEGIA BI-ETAPA PARA EL “LAYOUT” ÓPTIMO DE CELDAS DE MANUFACTURA
}

\section{A TWO-STAGE STRATEGY FOR THE OPTIMAL LAYOUT OF MANUFACTURING CELLS}

\author{
Nélida B. Camussi \\ Departamento de Ingeniería Industrial , Facultad de Ingeniería Química, \\ Universidad Nacional del Litoral \\ Santa Fe, Argentina \\ ncamussi@intec.unl.edu.ar
}

\begin{abstract}
RESUMEN: La disposición de los equipos en una planta, denominada simplificadamente "layout", resulta de sumo interés cuando se desea alcanzar metas específicas relativas a inventario en proceso, almacenamiento, fallas o interrupciones del proceso productivo, ordenamiento en la distribución de tareas, de recursos y del tráfico de operarios y dispositivos de traslado, defectos en la calidad, costos de manipulación de materiales, etc. En este trabajo se propone un algoritmo para la determinación de celdas y el "layout" óptimo de las mismas que consiste en dos etapas bien diferenciadas: primero se obtienen los grupos o celdas de máquinas (de equipos o, en forma más general, de elementos a ser agrupados) de manera tal que el costo del manejo de materiales inter-celdas sea mínimo y, en segundo lugar, teniendo como datos los grupos formados, se busca la mejor disposición de ellos entre sí y de las máquinas dentro de cada una de las celdas que haga más bajos tanto el costo de manipulación inter-celular como el intra-celular. Se presenta un estudio de caso a los efectos de comparación, comprobando así la eficiencia de la nueva propuesta.
\end{abstract}

Palabras clave: Tecnología de grupo. Celdas de Manufactura. Optimización Matemática.

ABSTRACT: Plant layout becomes especially interesting when specific goals relative to work -in- process, storage, machine failures or production process halting, tasks or resources distribution, manpower movement, quality problems, material handling costs and so on should be reached. In this work an algorithm for determining the cells and the optimal layout inside and between them is provided. The procedure consists of two stages: firstly, groups (cells) of machines are formed through a kind of quadratic assignment model in a way that minimizes the intercellular movement of parts. With the determination of groups in hand, the second stage seeks for the optimal layout design considering both within each cell and between cells costs of transportation of the parts (intra- and inter-cellular material handling costs, respectively). A case study is presented so as to compare previous results to those of the new proposal, just to prove the efficiency of the latter.

Keywords: Group Technology. Manufacturing Cells. Mathematical Optimization. 


\section{INTRODUCCIÓN}

Tecnología de grupo (TG) es un enfoque filosófico que intenta agrupar productos con características de diseño y/o manufactura similares (MITROFANOV, 1983). La manufactura celular (MC) está relacionada con la TG en cuanto que involucra el agrupamiento de máquinas basado en las partes manufacturadas por ellas. El principal objetivo de la MC es identificar celdas de máquinas y familias de partes simultáneamente y asignar familias de partes a celdas de máquinas de tal forma que minimice el movimiento intercelular de partes.

Para implementar satisfactoriamente el concepto de MC, los analistas deben desarrollar el layout de máquinas dentro de las celdas de manera de minimizar los costos de manejo de materiales inter- e intra-celular. MC es un concepto que ha sido aplicado exitosamente en los ambientes de manufactura y puede alcanzar beneficios significativos: reducción del tiempo de setup, del inventario en proceso, del costo de manejo de materiales, del costo de mano de obra directa e indirecta, mejoras en la calidad, en el fluir de los materiales, en la utilización del espacio, etc.

La principal diferencia entre un ambiente jobshop tradicional y uno de MC está en el agrupamiento y layout de las máquinas (BURDBIDGE, 1992). En el caso del ambiente jobshop las máquinas se agrupan sobre la base de sus similaridad funcionales. Por otra parte, en MC las máquinas se agrupan en celdas, con cada celda dedicada a la manufactura de una familia de partes específica y, generalmente, las máquinas en cada celda tienen funciones disimilares.

Además de minimizar el movimiento intercelular de partes (o familias de ellas), un diseñador de sistemas de MC debe ser capaz de considerar otras restricciones (SINGH, 1993). Por ejemplo, las familias de partes deben ser asignadas a celdas de máquinas de manera que la capacidad disponible de máquinas en cada celda no sea excedida, se deben satisfacer requerimientos de seguridad y tecnológicos relativos a la ubicación del equipamiento y los procesos, y el tamaño de las celdas y el número de celdas no debe exceder ciertos valores especificados por el usuario.

La mayoría de los esfuerzos en TG y en diseño de sistemas de MC ignoran algunas o todas estas restricciones y se enfocan solamente en la identificación de las celdas de máquinas y las correspondientes familias de partes. Lo típico es partir de la matriz de incidencia "partemáquina" (solo con ceros y unos) que con valor uno en la entrada $a_{i j}$, indica que la parte $i$ es procesada por la máquina $j$. Esta matriz indicadora puede modificarse para manejar otros elementos de información valiosa a usarse para identificar celdas de máquinas y familias de 
partes. Por ejemplo, dos columnas adicionales pueden crearse para indicar el número de partes a fabricarse y el tamaño de batch de cada una de ellas. De manera similar, la secuencia de máquinas visitadas por una parte puede registrarse en una matriz. La secuencia de operaciones para cada parte es un factor crítico en la identificación de celdas de máquinas.

Existen varias técnicas para abordar el problema. Entre los más relevantes están los basados en sistemas de clasificación y codificación usados en ambientes de manufactura que permiten a los usuarios agrupar las partes fácilmente en base a sus atributos, tales como forma, dimensiones, materiales componentes, tolerancia y requerimiento de operaciones.

También se destacan entre los enfoques planteados las técnicas de clustering que trata de encontrar los clusters o grupos similares a partir de los datos de la matriz indicadora de procesamiento. La técnica consiste, básicamente, en reacomodar filas y columnas de la matriz de incidencia hasta que una forma diagonal en bloques se pueda identificar. Tales técnicas sirven fundamentalmente para identificar celdas de máquinas, familias de partes correspondientes o ambas cosas. No tienen en cuenta restricciones de diseño ni factores de costo. Entre los algoritmos más comúnmente usados se puede nombrar a ROC (Rank Order Clustering), energía de enlace, enmascaramiento de filas y columnas (R\&CM), coeficiente de similaridad e inclusive un modelo con programación matemática (modelo de la p-mediana) (HERAGU, 1997).

Uno de las objetivos mejor estudiados en TG tiene que ver básicamente con: (1) identificar partes con requerimientos de procesamiento similares, (2) identificar el conjunto de máquinas que puedan procesar esas partes y (3) dedicar el conjunto de máquinas a la manufactura de las partes. El conjunto de máquinas forma una 'celda' o cluster de máquinas y el correspondiente conjunto de partes forma una familia de partes.

La identificación de los grupos de máquinas y las correspondientes familias de partes se basan solamente en si una máquina es requerida para realizar una o más operaciones sobre una parte. La base para el problema de identificación del cluster es dada la matriz de incidencia "parte-máquina", reacomodar las filas y columnas para tratar de crear una forma diagonal en bloques. En ese proceso, las técnicas no tienen en cuenta algunas restricciones de diseño del mundo real que deben ser incorporadas cuando se diseñan sistemas de MC. Por ejemplo, una compañía puede desear arreglar las máquinas en celdas y las partes en familias de partes para minimizar el número de viajes vinculados al manejo de materiales entre celdas.

O una compañía podría estar interesada en formar celdas de máquinas para la utilización de máquinas más eficiente en cada celda. Ninguna de las técnicas nombradas en el párrafo anterior puede abordar directamente estas últimas problemáticas (HERAGU, 1997). En este 
trabajo se pretende incorporar la etapa de layout intra e intercelular a partir del agrupamiento hecho en una primera etapa que consta de la aplicación de un nuevo modelo matemático (CAMUSSI, 2011) que agrupa las máquinas en celdas minimizando el costo del manejo de materiales entre ellas. Se presenta la aplicación del algoritmo bi-etapa a un estudio de caso planteado por Heragu (1997) y los resultados obtenidos se comparan con los reportados en esta última referencia.

\section{DESARROLLO DE LA PROPUESTA}

\subsection{Nomenclatura}

\subsubsection{Conjuntos}

$M$ : Máquinas (o cualquier elemento a ser agrupado en las celdas)

$C$ : Celdas

\subsubsection{Subíndices}

$m, m$ ’ máquina

$c, c^{\prime}:$ celda

\subsubsection{Escalares}

MinEC: mínimo número de elementos (máquinas) por celda

MaxEC: número máximo de elementos (máquinas) por celda

$N M n C$ : número mínimo de celdas $(\geq 2)$

$N M x C$ : número máximo de celdas

$K, M$ : constantes suficientemente grandes

DimHT: cota horizontal del terreno donde se ubicarán las celdas

DimVT: cota vertical del terreno donde se ubicarán las celdas

\subsubsection{Parámetros}

$N E_{m}$ : número de componentes del tipo de máquina $m$

$f_{m m}$ : matriz de flujos o frecuencia de movimiento de materiales desde la máquina $m$ hacia la $m$ ', con $m \neq m$ ' (al ser una matriz de flujos “desde-hacia”, no es necesariamente simétrica)

$a_{m m}$ : matriz de costo unitario del manejo de materiales desde la máquina $m$ hacia la máquina $m^{\prime}$, con $m \neq m^{\prime}$ (observación similar a la hecha para la matriz $f_{m m^{\prime}}$ )

$l h_{m}$ : longitud de la máquina $m$ en la dirección horizontal correspondiente a la orientación de referencia 
$l v_{m}$ : altura de la máquina $m$ en la dirección vertical correspondiente a la orientación de referencia

$d h_{m m}$ : mínima distancia (o pasillo) horizontal entre las máquinas $m$ y $m$ '

$d v_{m m}$ : mínima distancia (o pasillo) vertical entre las máquinas $m$ y $m$ '

\subsubsection{Variables}

$y_{m c}$ : binaria que vale 1 cuando la máquina $m$ debe ubicarse en la celda de manufactura $c$

$x_{c}$ : binaria que vale 1 si la celda $c$ tiene al menos un elemento en ella

$L H C_{c}$ : límite inferior horizontal de la celda $c$

$U H C_{c}$ : límite superior horizontal de la celda $c$

$L V C_{c}$ : límite inferior vertical de la celda $c$

$U V C_{c}$ : límite superior vertical de la celda $c$

$r_{m}$ : binaria que con valor 1 indica que la máquina $m$ debe ser rotada $90^{\circ}$ respecto de su orientación de referencia; con valor 0 , la mantiene.

lhvble $_{m}$ : longitud horizontal efectiva de la máquina $m$

$l_{v} v b_{e_{m}}$ : longitud vertical efectiva de la máquina $m$

$c x_{m}$ : coordenada horizontal del centro de gravedad de la máquina $m$

$c y_{m}$ : coordenada vertical del centro de gravedad de la máquina $m$

$\operatorname{cg} x_{c}$ : coordenada horizontal del centro de gravedad de la celda $c$

$c g y_{c}$ : coordenada vertical del centro de gravedad de la celda $c$

$p_{m m}$ : variable binaria que cuando vale 1 indica que la restricción de no-solapamiento de máquinas $m$ y $m$ ' se cumple en la dirección vertical; caso contrario, el no solapamiento se da en la dirección horizontal.

$c p_{c c}$ : variable binaria que cuando vale 1 indica que la restricción de no-solapamiento de celdas $c$ y c' se cumple en la dirección vertical; caso contrario, el no solapamiento se da en la dirección horizontal.

\subsection{Primera etapa del algoritmo}

En primer lugar se busca generar la partición del total de máquinas en celdas o grupos de manera de minimizar el costo del movimiento de materiales entre celdas. El modelo matemático propuesto para este fin es el propuesto en Camussi (2011) que consiste en un modelo mixto entero no-lineal (MINLP) de tipo cuadrático, cuyas expresiones algebraicas constitutivas se dan brevemente a continuación. 
Cada máquina debe estar en alguna celda activa. Se entiende como celda activa a aquella que alberga al menos un elemento (máquina, estación de trabajo, equipo, instalación, etc.). Una celda activa significa (Ecuación 1) que la variable $x_{c}$ toma el valor uno, mientras que una celda inactiva es denotado con un valor de $x_{c}$ nulo.

$$
\sum_{c=1}^{c=C} y_{m c}=1 \quad \forall m \in M
$$

Si una celda $c$ se activa, debe necesariamente albergar al menos una máquina $m$ (Ecuación 2).

$$
\sum_{m=1}^{m=M} y_{m c} \geq x_{c} \quad \forall c \in C
$$

Si la máquina $m$ es asignada a la celda $c$ (Ecuación 3 ), entonces la celda $c$ debe activarse.

$$
x_{c} \geq y_{m c} \quad \forall m \in M, \forall c \in C
$$

En cada celda activa debe haber un número mínimo y uno máximo de elementos, denotados MinEC y MaxEC, respectivamente (Ecuación 4 y 5).

$$
\begin{array}{ll}
\sum_{m=1}^{m=M} N E_{m} y_{m c} \geq \operatorname{MinEC} x_{c} & \forall c \in C \\
\sum_{m=1}^{m=M} N E_{m} y_{m c} \leq M \operatorname{axEC} x_{c} & \forall c \in C
\end{array}
$$

Existe un número mínimo y uno máximo de celdas, denotados $N M n C$ y $N M x C$ (Ecuación 6 y 7), respectivamente.

$$
\begin{aligned}
& \sum_{c=1}^{c=C} x_{c} \geq N M n C \\
& \sum_{c=1}^{c=C} x_{c} \leq N M x C
\end{aligned}
$$

La función objetivo (Ecuación 8) se expresa como una función cuadrática que busca minimizar el costo total del manejo de materiales entre las celdas generadas.

$$
z=\sum_{m=1}^{m=M} \sum_{c=1}^{c=c} \sum_{\substack{m^{\prime}=1 \\ m \\ m \neq m}}^{m^{\prime}=M} \sum_{\substack{c^{\prime}=1 \\ c^{\prime} \neq c}}^{c^{\prime}=c} a_{m m}, f_{m m}, y_{m c} y_{m^{\prime} c^{\prime}}
$$

Todas las variables de decisión del problema son binarias, excepto $z$ que es real y libre (Ecuación 9).

$$
\begin{aligned}
& y_{m c}, x_{c} \in\{0,1\} \quad \forall m \in M, c \in C \\
& -\infty \leq z \leq \infty
\end{aligned}
$$

El modelo matemático se expresa entonces como la minimización de la función objetivo dada en (Ecuación 8) sujeto a las restricciones (1)-(7) y (9). Para su resolución se puede apelar a los programas de optimización Dicopt y Baron para modelos MINLPs, ambos disponibles 
con el lenguaje de modelado GAMS (BROOKE, 2006). Ambos resolvedores sirven para la optimización matemática mixta entera no-lineal, pero la diferencia es que Dicopt suministra soluciones sin garantizar la optimalidad global, excepto que el programa sea convexo, y reporta, generalmente, óptimos locales. En cambio, Baron es un motor que asegura la optimalidad global de los MINLPs. Para un mismo problema, las corridas con el primero consumen, la mayoría de las veces, menos tiempo que aquellas con el segundo resolvedor.

\subsection{Segunda etapa del algoritmo}

Una vez determinado tanto el número óptimo de celdas como las máquinas que pertenecen a cada una de ellas, se procede a tratar de establecer la disposición (denotada también como layout) óptima tanto de los equipos dentro como entre ellas mismas.

El modelo que se plantea en esta segunda etapa se basa en uno de los modelos matemáticos que aparecen como ABSMODEL de Heragu (1997), incorporándose ahora algunas modificaciones que permiten, por ejemplo, que el modelo decida la orientación óptima de las máquinas componentes, como así también los valores óptimos de los límites inferior y superior, horizontal y vertical, de cada una de las celdas, que, a su vez, se supondrán de forma rectangular.

También, vale aclarar que tanto aquel modelo básico como el ampliado en este trabajo, son válidos para máquinas (instalaciones en general) de forma rectangular que no poseen, necesariamente, igual área. Las variables fundamentales del modelo son las coordenadas horizontal y vertical de los centros de gravedad de las máquinas y los de las celdas. Es, por lo tanto, un modelo bidimensional, que tiene en cuenta el largo y el alto de las instalaciones a disponer. Para entender gráficamente las variables de decisión y las restricciones planteadas se remite a la Figura 1. Las siglas LRH y LRV que se ven sobre ellas representan las "líneas de referencia horizontal" y "línea de referencia vertical", respectivamente.

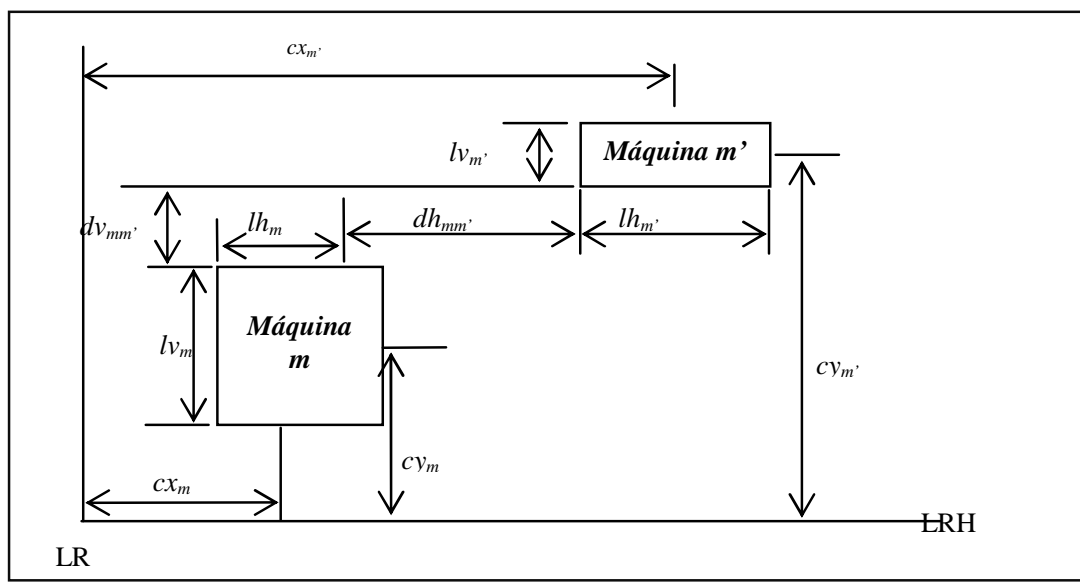


Figura 1 - Ilustración de los parámetros y algunas de las variables de decisión correspondientes a un modelo de layout bidimensional con máquinas de forma rectangular de distinta área

Las restricciones (Ecuación 10 y 11) aseguran que las máquinas no se "solapen” en la dirección horizontal o vertical y que se satisfagan las eventuales separaciones mínimas de pasillo en una cualquiera o en ambas direcciones. La restricción solo se plantea para $m<m$ ' por la simetría de la función "valor absoluto". Basta con que una de las dos se cumpla en forma activa para una de las dos dimensiones para garantizar que los centros de gravedad de las máquinas mantienen las correspondientes distancias (para que los espacios que ocupan no se superpongan) en virtud de la suposición de forma rectangular (o circunscripción en un rectángulo) de las máquinas.

$$
\begin{gathered}
\left|c x_{m}-c x_{m^{\prime}}\right|+M p_{m m^{\prime}} \geq 0.5\left(\text { lhvble }_{m}+\text { lhvble } e_{m^{\prime}}\right)+d h_{m m^{\prime}} \\
\forall m, m^{\prime}: m<m^{\prime} \\
\left|c y_{m}-c y_{m^{\prime}}\right|+M\left(1-p_{m m^{\prime}}\right) \geq 0.5\left(\text { lvvble }_{m}+\text { lvvble }_{m^{\prime}}\right)+d v_{m m^{\prime}} \\
\forall m, m^{\prime}: m<m^{\prime}
\end{gathered}
$$

Las máquinas pertenecientes a cada celda (cuyos componentes se definieron en la primera etapa) no deben exceder los límites de la misma (Ecuación 12, 13 14, y 15).

$$
\begin{array}{ll}
c x_{m} \geq \text { LHC }_{c}+0.5 \text { lhvble }_{m} & \forall m, c: m \in \text { celda } c \\
c x_{m} \leq U H C_{c}-0.5 \text { lhvble }_{m} & \forall m, c: m \in \text { celda } c \\
c y_{m} \geq L V C_{c}+0.5 \text { lvvble }_{m} & \forall m, c: m \in \text { celda } c \\
c y_{m} \leq U V C_{c}-0.5 \text { lvvble }_{m} & \forall m, c: m \in \text { celda } c
\end{array}
$$

La longitud horizontal y vertical de cada máquina $m$ dependerá de si la máquina debe ser rotada o no respecto de una orientación de referencia dada.

$$
\begin{array}{ll}
\text { lhvble }_{m}=r_{m} l v_{m}+\left(1-r_{m}\right) l h_{m} & \forall m \in M \\
\text { lvvble }_{m}=r_{m} l h_{m}+\left(1-r_{m}\right) l v_{m} & \forall m \in M
\end{array}
$$

Las celdas tienen cotas inferior y superior en la dirección horizontal dadas por $L H C_{c} \mathrm{y}$ $U H C_{c}$, respectivamente, y cotas inferior y superior vertical dadas por $L V C_{c}$ y $U V C_{c}$, respectivamente. Es decir que el ancho de una celda está dado por $U H C_{c}-L H C_{c}$ y la altura está dada por $U V C_{c}-L V C_{c}$ (Figura 2). 


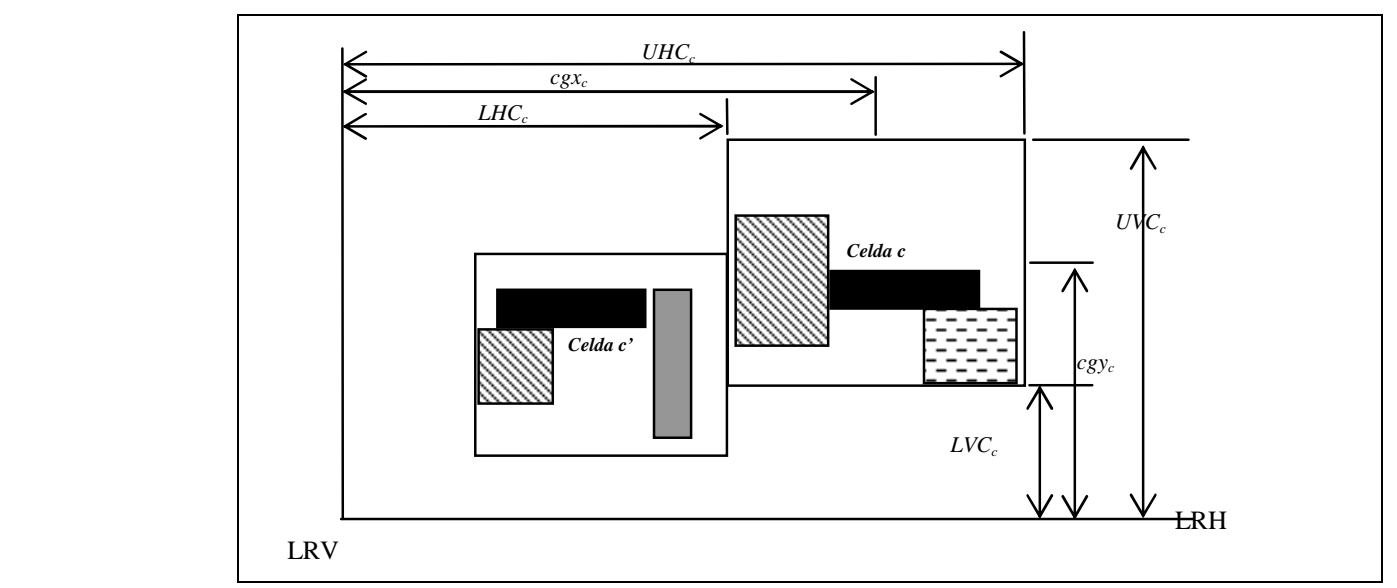

Figura 2 - Variables de decisión en el problema de "layout” bidimensional de las celdas de manufactura

Análogamente a las máquinas, las restricciones (Ecuación 18 y 19) buscan garantizar la no-superposición de las celdas (Figura 2).

$$
\begin{gathered}
\left|c g x_{c}-c g x_{c^{\prime}}\right|+M c p_{c c^{\prime}} \geq \\
\geq 0.5\left(U H C_{c}-L H C_{c}+U H C_{c^{\prime}}-L H C_{c^{\prime}}\right) \\
\forall c, c^{\prime}: c<c^{\prime} \\
\left|c g y_{c}-c g y_{c^{\prime}}\right|+M\left(1-c p_{c c^{\prime}}\right) \geq \\
\geq 0.5\left(U V C_{c}-L V C_{c}+U V C_{c^{\prime}}-L V C_{c^{\prime}}\right) \\
\forall c, c^{\prime}: c<c^{\prime}
\end{gathered}
$$

Con las Ecuación (20) y (21) se calcula el centro de gravedad de cada celda dado por el punto medio del área rectangular determinado para cada una de ellas.

$$
\begin{array}{ll}
c g x_{c}=0.5\left(U H C_{c}+L H C_{c}\right) & \forall c \in C \\
c g y_{c}=0.5\left(U V C_{c}+L V C_{c}\right) & \forall c \in C
\end{array}
$$

El objetivo dado por (Ecuación 22) es minimizar el costo de la manipulación de materiales a través de los distintos recorridos entre máquinas y entre celdas que se realizan para la producción de las partes. Se supone que tales trayectos se miden según la distancia de Manhattan entre los centros de gravedad. La idea es que aquellas máquinas (celdas) que tengan un costo de tráfico muy alto se ubiquen en el layout lo más cercanamente posible.

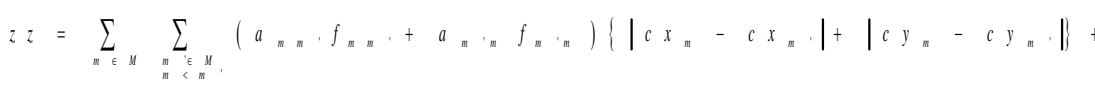

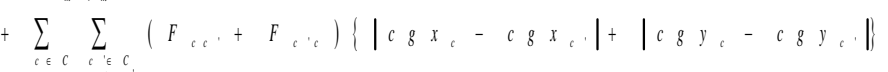

Donde $F_{c c^{\prime}}=\sum_{\substack{m \in M \\ m \in c}} \sum_{\substack{m^{\prime} \in M \\ m \in c^{\prime}}} a_{m m^{\prime}} f_{m m^{\prime}} \quad \forall c, c^{\prime} \in C: c \neq c^{\prime}($ Ecuación 23) 
$F_{c c}$ 'representa la matriz de costo del flujo inter-celular (que no es necesariamente simétrica) y cuya sumatoria de elementos coincide con el valor óptimo obtenido en la primera etapa. Tampoco es necesariamente simétrica la matriz de frecuencia de viajes entre máquinas, $f_{m m}$ ' ni la de costos, $a_{m m}$, por eso, y dada la simetría de la función "valor absoluto", aparecen en la Ecuación (22) los términos en ambos sentidos, $m m^{\prime}$ y m’m ó $c c^{\prime}$ y c'c. Esta función objetivo busca que las máquinas y celdas estén lo más cercanas posibles cuando el manejo de materiales entre ellas sea muy costoso, sin especificar si están una a la derecha, izquierda, arriba o debajo de la otra.

Este modelo de no solapamiento con valores absolutos se puede modelar en forma análoga a la aquí expresada usando variables de decisión tales como "estar a la izquierda de", "estar a la derecha de", "estar arriba de" y "estar debajo de", pero el modelo crece considerablemente en número de restricciones como de variables binarias, por lo tanto no se hará un planteo explícito de esa propuesta en este trabajo.

Otra cuestión que es importante notar en la Ecuación (22) es que el primer bloque de términos representa el costo real del manejo de materiales entre todas las máquinas (tanto las de una misma celda, como de distintas celdas), mientras que el segundo bloque de términos tiene coeficientes de costo que representan los costos globales del manejo de materiales entre celdas, cuando en realidad los manejos se hacen entre máquinas y que efectivamente ya han sido considerados en el primer bloque. Sin embargo, se agrega igualmente el segundo bloque pues el objetivo que se pretende es el layout óptimo dentro de las celdas y entre las celdas en forma simultánea.

Se establecen cotas de terreno para todo el conjunto de las celdas, a través de Ecuación 24 y 25 .

$$
\begin{array}{ll}
U H C_{c} \leq \operatorname{dim} H T & \forall c \in C \\
U V C_{c} \leq \operatorname{dim} V T & \forall c \in C
\end{array}
$$

Las características de cada variable de decisión se expresan en Ecuación (26).

$$
\begin{aligned}
& c x_{m} \geq 0, c y_{m} \geq 0, \text { cgx }_{c} \geq 0, \text { cgy }_{c} \geq 0, \text { lhvble }_{m} \geq 0, \text { lvvble }_{m} \geq 0, \\
& L H C_{c} \geq 0, U H C_{c} \geq 0, L V C_{c} \geq 0, U V C_{c} \geq 0 \quad \forall m \in M, c \in C \\
& r_{m}, p_{m m^{\prime}}, c p_{c c^{\prime}} \in\{0,1\} \quad \forall m \in M, c \in C \\
& -\infty \leq z z \leq \infty
\end{aligned}
$$


El modelo matemático que minimiza la Ecuación (22) sujeta a las restricciones (10)(26) devuelve los valores óptimos de las variables de decisión detalladas en Ecuación (26) y son las que minimizan el costo del movimiento de materiales intra- e inter-celular.

Debido a la existencia de la función "valor absoluto" afectando a variables del problema, se recurre a un artificio matemático que genera un modelo equivalente, aunque con más variables y restricciones. Se hará el detalle solo para el $\left|c x_{m}-c x_{m},\right|$ (Ecuación 27 y 28) a efectos ilustrativos; para el resto de los valores absolutos se trabaja en forma similar. Se reemplaza la función "valor absoluto" por la suma de dos funciones según se detalla a continuación:

$$
\begin{gathered}
c x_{m m^{\prime}}^{+}=\left\{\begin{array}{c}
\left(c x_{m}-c x_{m^{\prime}}\right) \text { si }\left(c x_{m}-c x_{m^{\prime}}\right)>0 \\
0 \text { en cualquier otro caso }
\end{array}\right. \\
c x_{m m^{\prime}}^{-}=\left\{\begin{array}{c}
\left(c x_{m^{\prime}}-c x_{m}\right) \text { si }\left(c x_{m}-c x_{m^{\prime}}\right) \leq 0 \\
0 \text { en cualquier otro caso }
\end{array}\right.
\end{gathered}
$$

A partir de las definiciones dadas en (27) y (28), es fácil deducir que (Ecuación 29 y 30):

$$
\begin{gathered}
\left|c x_{m}-c x_{m^{\prime}}\right|=c x_{m m^{\prime}}^{+}+c x_{m m^{\prime}}^{-} \quad \forall m, m^{\prime} \in M: m<m^{\prime} \\
c x_{m}-c x_{m^{\prime}}=c x_{m m^{\prime}}^{+}-c x_{m m^{\prime}}^{-} \quad \forall m, m^{\prime} \in M: m<m^{\prime}
\end{gathered}
$$

Una de las condiciones que deben cumplir las funciones sustitutas del "valor absoluto" es la dada por Ecuación (31):

$$
c x_{m m^{\prime}}^{+} \cdot x_{m m^{\prime}}^{-}=0 \quad \forall m, m^{\prime} \in M: m<m^{\prime}
$$

Si se incorporase esta restricción en el modelo matemático el problema se volvería no lineal y bastante arduo de resolver. Sin embargo, hay otra forma de lograr el mismo efecto buscado en Ecuación (31) con restricciones lineales, aunque involucrando un nuevo grupo de variables binarias y es a través del agregado de las restricciones (32) y (33).

$$
\begin{gathered}
c x_{m m^{\prime}}^{+} \leq K \text { binc }_{m m^{\prime}} \quad \forall m, m^{\prime} \in M: m<m^{\prime} \\
c x_{m m^{\prime}}^{-} \leq K\left(1-\text { binc }_{m m^{\prime}}\right) \quad \forall m, m^{\prime} \in M: m<m^{\prime}
\end{gathered}
$$

De resultas de estos cambios, se obtiene un modelo matemático mixto-entero lineal (MILP) con más restricciones y variables continuas y binarias que es equivalente al planteado en este trabajo usando las expresiones con "valor absoluto". 


\section{ESTUDIO DE CASO}

El estudio de caso presentado es un proyecto industrial que aparece en el capítulo 9 de Heragu (1997). Mediquip Manufacturer Inc. tiene un "layout" de proceso en el cual ocho máquinas que pertenecen a cinco tipos distintos se acomodan como se ve en la Figura 3. Por diversas razones, que incluyen cambios drásticos en la mezcla de productos y los volúmenes, el "layout" actual ha derivado en problemas como gran congestión de tráfico, falta de control en el sistema, baja de la motivación en los empleados e incremento en los tiempos y en los costos de manejo de materiales.

El grupo de ingeniería industrial en Mediquip sugiere fuertemente reagrupar las máquinas en celdas (de manera que cada una de estas últimas se dedique a la manufactura de un conjunto de partes con características de procesamiento similares) que aliviará mucho de los problemas. Como se mencionó, el layout actual se ve en la Figura 3 y está conformado por ocho equipos, los tipos (y cantidad existente) de cada uno son: 1204 (1), 2014 (1), 2008 (1), 2023 (1), 2029 (2) y 2030 (2).

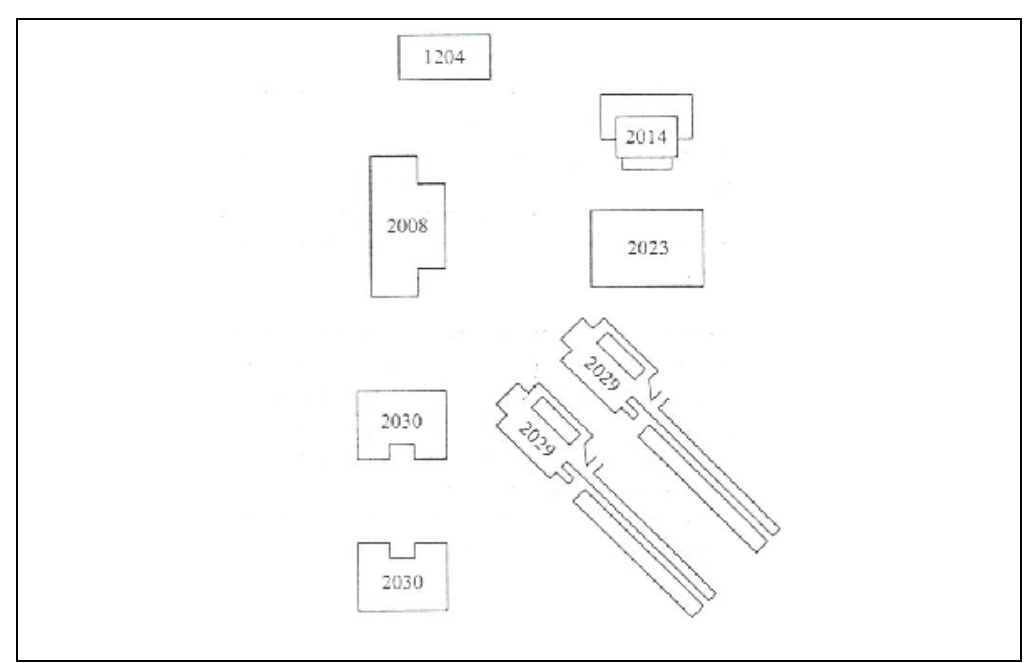

Figura 3 - Disposición actual de máquinas en la planta Fuente: Heragu (1997)

En la Tabla 1 se despliegan algunos datos fundamentales para el análisis que debe realizarse, a saber: información de "routing" de 20 partes, tiempos de setup, tiempos de procesamiento, tamaño de batch y demanda anual. La secuencia de procesamiento (o “routing") de cada producto, debe leerse por filas. Así, por ejemplo, el producto 1 pasa en primer lugar por la máquina 1204 y luego por la 2008. En el caso del producto 4, arranca por el equipo 2008, en segundo lugar va al 2023, en tercer lugar vuelve al 2008 (con distintos parámetros de operación) y finalmente pasa por el 2014. 
Tabla 1 - Datos relevantes de las partes fabricadas

\begin{tabular}{|c|c|c|c|c|c|}
\hline $\begin{array}{l}\text { Número de } \\
\text { identificación } \\
\text { de la parte }\end{array}$ & $\begin{array}{c}\text { Demanda } \\
\text { anual (unid.) }\end{array}$ & $\begin{array}{l}\text { Tamaño de } \\
\text { batch (unid.) }\end{array}$ & $\begin{array}{l}\text { Nombre de } \\
\text { máquina }\end{array}$ & $\begin{array}{l}\text { Tiempo de } \\
\text { setup por } \\
\text { batch (hrs) }\end{array}$ & $\begin{array}{c}\text { Tiempo de } \\
\text { procesamiento } \\
\text { por batch (hrs) }\end{array}$ \\
\hline \multirow[t]{2}{*}{1} & 220 & 10 & 1204 & 3.00 & 3.64 \\
\hline & & & 2008 & 3.00 & 5.98 \\
\hline \multirow[t]{3}{*}{2} & 500 & 10 & 1204 & 5.00 & 2.00 \\
\hline & & & 2008 & 2.25 & 1.00 \\
\hline & & & 2014 & 0.50 & 6.50 \\
\hline \multirow[t]{2}{*}{3} & 500 & 10 & 1204 & 4.00 & 2.00 \\
\hline & & & 2008 & 1.00 & 3.00 \\
\hline \multirow[t]{4}{*}{4} & 500 & 10 & 2008 & 1.50 & 3.00 \\
\hline & & & 2023 & 0.50 & 8.27 \\
\hline & & & 2008 & 0.00 & 2.00 \\
\hline & & & 2014 & 2.00 & 2.00 \\
\hline \multirow[t]{3}{*}{5} & 220 & 10 & 2008 & 1.00 & 2.00 \\
\hline & & & 2014 & 1.00 & 6.86 \\
\hline & & & 2023 & 1.00 & 10.00 \\
\hline \multirow[t]{2}{*}{6} & 120 & 10 & 2030 & 0.50 & 84.58 \\
\hline & & & 2023 & 1.00 & 26.78 \\
\hline \multirow[t]{2}{*}{7} & 700 & 10 & 2008 & 1.00 & 1.00 \\
\hline & & & 2023 & 1.00 & 1.00 \\
\hline \multirow[t]{3}{*}{8} & 900 & 10 & 2030 & 0.75 & 1.25 \\
\hline & & & 2014 & 0.50 & 1.00 \\
\hline & & & 2029 & 0.00 & 0.31 \\
\hline \multirow[t]{2}{*}{9} & 200 & 10 & 2029 & 8.00 & 65.00 \\
\hline & & & 2014 & 0.00 & 2.23 \\
\hline \multirow[t]{2}{*}{10} & 250 & 10 & 2030 & 0.50 & 5.00 \\
\hline & & & 2008 & 1.00 & 7.00 \\
\hline \multirow[t]{2}{*}{11} & 400 & 10 & 2029 & 8.00 & 55.00 \\
\hline & & & 2014 & 1.00 & 2.67 \\
\hline \multirow[t]{2}{*}{12} & 300 & 10 & 1204 & 2.89 & 8.68 \\
\hline & & & 2008 & 1.00 & 8.00 \\
\hline \multirow[t]{3}{*}{13} & 250 & 10 & 2029 & 0.00 & 8.55 \\
\hline & & & 2030 & 0.00 & 19.37 \\
\hline & & & 2014 & 1.50 & 2.80 \\
\hline \multirow[t]{3}{*}{14} & 600 & 10 & 2029 & 0.00 & 7.53 \\
\hline & & & 2030 & 1.00 & 3.00 \\
\hline & & & 2014 & 0.75 & 1.00 \\
\hline \multirow[t]{2}{*}{15} & 450 & 10 & 2030 & 1.00 & 2.91 \\
\hline & & & 2030 & 1.50 & 3.83 \\
\hline \multirow[t]{2}{*}{16} & 500 & 10 & 1204 & 4.00 & 3.64 \\
\hline & & & 2008 & 0.75 & 3.00 \\
\hline \multirow[t]{2}{*}{17} & 400 & 10 & 2030 & 0.00 & 2.50 \\
\hline & & & 2023 & 0.50 & 2.00 \\
\hline \multirow[t]{3}{*}{18} & 300 & 10 & 2029 & 0.00 & 12.01 \\
\hline & & & 2030 & 0.00 & 7.59 \\
\hline & & & 2023 & 0.75 & 14.50 \\
\hline \multirow[t]{3}{*}{19} & 10 & 10 & 2029 & 0.00 & 23.25 \\
\hline & & & 2030 & 0.00 & 23.63 \\
\hline & & & 2008 & 0.00 & 42.67 \\
\hline \multirow[t]{3}{*}{20} & 250 & 10 & 2029 & 0.00 & 7.28 \\
\hline & & & 2030 & 0.00 & 23.00 \\
\hline & & & 2023 & 1.00 & 2.00 \\
\hline
\end{tabular}

La Tabla 2 muestra los dispositivos usados en el manejo de materiales entre máquinas. Aunque el costo exacto de manipular una carga unitaria a lo largo de una unidad de distancia 
sobre cada tipo de dispositivo de manejo de material es difícil de obtener, se sabe que los vehículos guiados automáticamente son el triple más caro (por carga unitaria por unidad de distancia) que el transporte manual a través de carros y que hacerlo a través de furgones es el doble de caro que el transporte manual para este ejemplo.

Tabla 2 - Dispositivos usados para el manejo de materiales

\begin{tabular}{c|cc}
\hline Desde máquina (s) & Hacia máquina (s) & Dispositivo usado \\
\hline 1204 & Todas las otras & Furgón \\
2008 & Todas las otras & Manualmente con carro \\
2014 & Todas las otras & Vehículo guiado \\
& & automáticamente (AGV) \\
2023 & 1204,2008 & Manualmente con carro \\
2023 & 2029 & Furgón \\
2023 & 2014,2030 & Vehículo guiado \\
& & automáticamente (AGV) \\
2029 & Todas las otras & Manualmente con carro \\
\hline $\mathbf{2 0 3 0}$ & Todas las otras & Furgón \\
\hline
\end{tabular}

La Tabla 3 dimensiones horizontal (longitud) y vertical (altura) de las máquinas correspondientes a la orientación de referencia (en pies).

Tabla 3 - Dimensiones horizontal (longitud) y vertical (altura) de las máquinas correspondientes a la orientación de referencia (en pies)

\begin{tabular}{c|cc}
\hline Máquina & Longitud & Altura \\
\hline 1204 & 20 & 10 \\
2008 & 20 & 15 \\
2014 & 15 & 10 \\
2023 & 25 & 20 \\
2029 & 45 & 10 \\
\hline $\mathbf{2 0 3 0}$ & 20 & 15 \\
\hline
\end{tabular}

La gerencia de producción de Mediquip desea que no haya más de tres máquinas por celda debido a la carencia de personal entrenado. No hay ninguna restricción de adyacencia especial entre cualquier par de máquinas. Si hay disponible más de una unidad de un tipo de máquina para procesamiento, se supone que cada unidad de equipamiento tiene la misma probabilidad de procesar las partes que deben pasar por ese tipo de máquina. Lo primero que se debe determinar al resolver el problema de TG y el de "layout" es si hay suficiente capacidad para procesar el volumen de partes proyectado. A partir de la demanda anual y el tamaño de batch, se puede fácilmente determinar el número de batches de cada parte que deben ser procesados anualmente dividiendo la demanda por el tamaño de batch. Luego usando los tiempos de setup y procesamiento por batch, se puede calcular el tiempo requerido por cada tipo de máquina para procesar cada una de las 20 partes.

Heragu (1997) supone que está incurriendo en un tiempo de setup por batch, aunque aclara que en la práctica dos ó más batches pueden ser producidos consecutivamente, 
requiriendo por lo tanto, menos setups). Sumando esos valores da las horas totales requeridas por cada tipo de máquina. Luego, dividiendo esos valores por el tiempo disponible (que se supone de 40 horas semanales de operación, 50 semanas al año con una eficiencia del 90\%, que da 1800 horas) da una estimación del número de máquinas de cada tipo requeridas. Se anticipa una utilización de máquina menor al 90\% debido a indisponibilidad de materia prima, equipamiento u operadores de máquinas o de manejo de materiales, paradas de máquina, etc.).

Bajo estas suposiciones, se encuentra que se necesitan tres unidades de la máquina de tipo 2029, dos unidades de tipo 2030 y una unidad de los otros tipos para poder cumplir con lo proyectado, es decir, nueve equipos en total. Se sabe que actualmente hay ocho equipos (Figura 3), de modo que se debe comprar una unidad adicional de máquina del tipo 2029, elevándose de ocho a nueve el número total de equipos a disponer en ese sector de planta. Además existen restricciones de pasillo de 2 pies a cada lado de las máquinas, por lo que $d h_{m m^{\prime}}=4$ y $d v_{m m}=4, \forall m, m^{\prime}$, con $m \neq m^{\prime}$. No existen restricciones acerca de las dimensiones del terreno.

Heragu (1997) se reporta su resolución usando un software disponible denominado GTLAYPC.EXE, de naturaleza interactiva, que identifica no solo las celdas sino también su "layout" y el "layout" de máquinas dentro de cada celda. El programa se basa en un método de tres etapas. En la primera las máquinas se agrupan en celdas (proponiendo agrupamientos de máquinas en forma exhaustiva) y las correspondientes partes en familias de partes (con el algoritmo R\&CM). En la segunda etapa, el "layout" de máquinas dentro de cada celda se determina mientras que en la tercera se hace lo propio pero con las celdas (ambas usando heurísticas como 2-opt, simulated annealing). La Figura 4 muestra el gráfico de la disposición obtenida tras su aplicación. El costo óptimo reportado es 58511.55 .

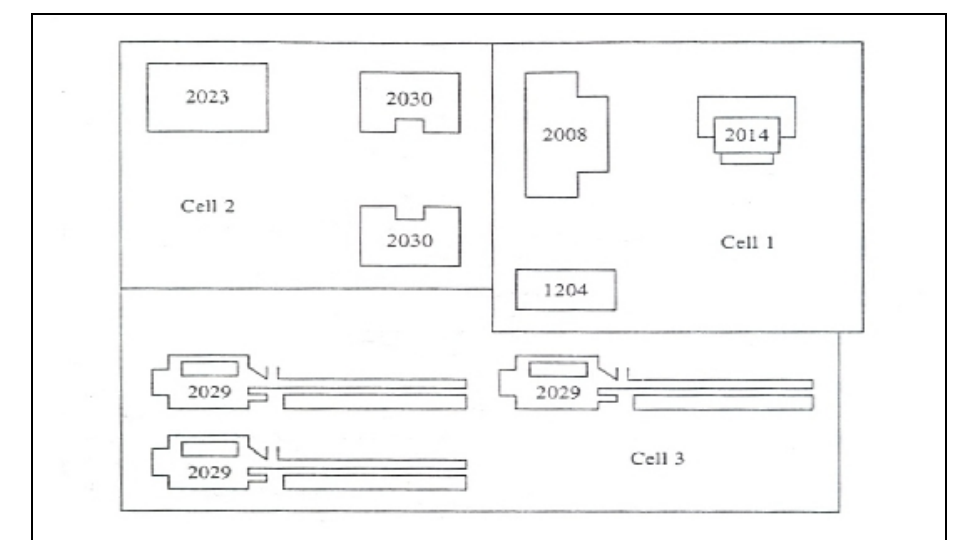

Figura 4 - Solución reportada con un software de uso interactivo que emplea heurísticos Fuente: Heragu (1997) 
Aplicando la propuesta hecha en el presente trabajo, en la primera etapa del algoritmo se plantea un modelo matemático MINLP con 63 restricciones, 42 variables binarias y 1 continua, que se resolvió con el resolvedor BARON (BROOKE, 2006) (disponible con la plataforma de modelado GAMS) en 0.89 seg de $C P U$ corrido sobre una computadora $H P$ Z600 con un procesador Intel Xeon de $2.67 \mathrm{Ghz}$ y $16 \mathrm{~GB}$ de $R A M$ con valor de costo intercelular óptimo igual a 819. Se formaron tres celdas: La primera integrada con 2014 y las dos 2030; la segunda, con 1204, 2008 y 2023 y una tercera formada con los tres equipos 2029. Si se calcula la suma de frecuencias correspondiente a la solución graficada en la Figura 4, su valor es de 662 y su costo total da 1083, mientras que la totalidad de flujo para agrupamiento obtenido con el MINLP es de 506 con un costo total de 819.

Con esos resultados como datos, se pasó a la aplicación de la segunda etapa del algoritmo que resultó en un modelo matemático MILP con 374 restricciones, 212 variables continuas, 126 variables binarias que alcanzó el valor óptimo de 48992 usando el resolvedor Gurobi (también disponible con GAMS), en 84.34 segundos de $C P U$ con una computadora HP Z600 con un procesador Intel Xeon de $2.67 \mathrm{Ghz}$ y $16 \mathrm{~GB}$ de RAM. En la Figura 5 se presenta un esquema de la disposición óptima obtenida.

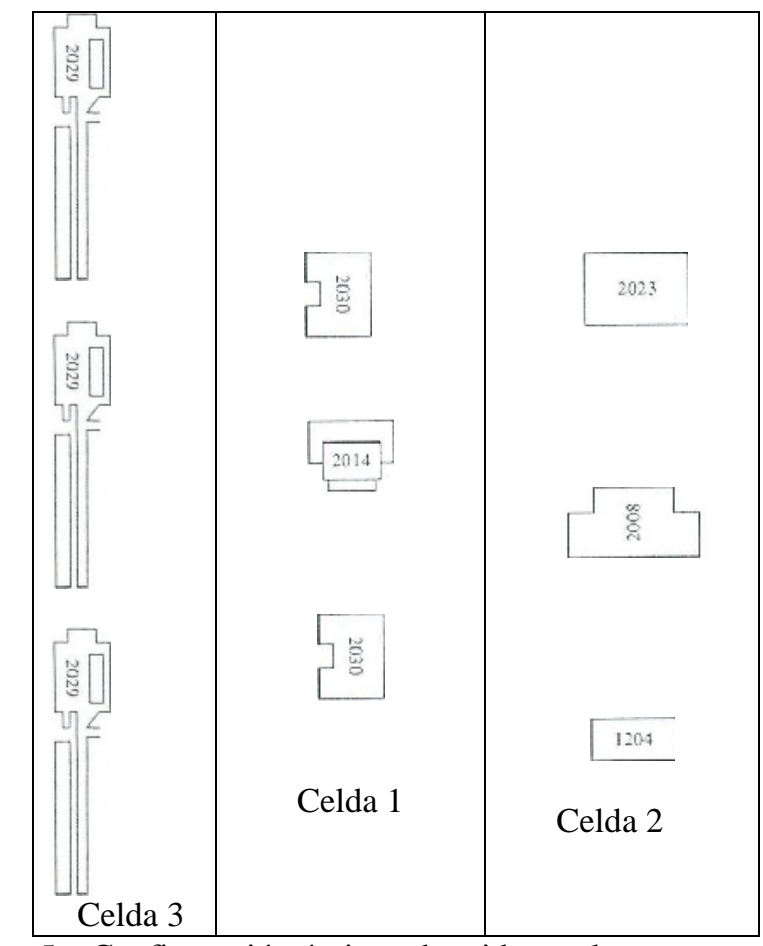

Figura 5 - Configuración óptima obtenida con la nueva propuesta

El ahorro en costos correspondiente es del 16.3\%. El modelo dispuso además la rotación del equipo de tipo 2008, de las tres unidades 2029 y de las dos 2030. El resto mantuvo las orientaciones de referencia. Los límites inferiores horizontales óptimos de las celdas $\mathrm{C}_{1}, \mathrm{C}_{2} \mathrm{y}$ 
$\mathrm{C}_{3}$, son 14, 29 y 0, respectivamente. Los límites superiores horizontales óptimos son 29, 58 y 14, también respectivamente. Los límites inferiores verticales óptimos son nulos para las tres celdas y los límites superiores verticales valen 143 para los tres grupos.

\section{CONCLUSIONES}

Se ha propuesto un algoritmo que consta de dos pasos obteniendo por un lado el agrupamiento de las máquinas, sin considerar la disposición de las mismas dentro de cada grupo o cluster, buscando reducir al máximo el costo del manejo de materiales entre grupos. A posteriori, con los resultados de "clusterización" obtenidos en la primera etapa, se procede a buscar la disposición óptima de las máquinas dentro de cada celda y además la ubicación y dimensiones óptimas de cada cluster que minimice los costos de movimientos intra- e intercelular. Se aplicó a un estudio de caso reportado en la literatura y se obtuvieron mejores soluciones en tiempos de CPU para su resolución bajos a pesar de ser un modelo MINLP seguido de uno MILP, lo cual lo hace competitivamente eficiente frente al procedimiento usado anteriormente consistente en combinaciones de heurísticos que suelen ser muy rápidos.

\section{REFERENCES}

BROOKE, A.; KENDRICK, D; MEERAUS, A.; RAMAN, R. GAMS: a users guide. GAMS Development Corporation. Washington, 2006.

BURDBIDGE, J. L. Change to group technology: process organization is obsolete. International Journal of Production Research, v. 30, n. 5, p. 1209-1219, 1992.

CAMUSSI, N. B. Modelo de programación matemática para la determinación cuasi-óptima de celdas de manufactura. In: Actas de XXIV Encuentro Nacional de Docentes en Investigación Operativa (ENDIO) y XXII Escuela de Perfeccionamiento en Investigación Operativa (EPIO), Río Cuarto, 2011.

HERAGU, S. Facilities design. PWS Publishing Company, 1997.

MITROFANOV, S. P. Group Technology in Industry. v.1-2. Leningrado: Mashinostroienie, 1983.

SINGH, N. Design of cellular manufacturing systems. European Journal of Operational Research, v. 69, n. 3, p. 284-291, 1993. 